\title{
Modelando um sistema tutor multiagentes para auxiliar na aprendizagem da matemática
}

\author{
Modeling a multi-agent system tutor to assist in learning mathematics
}

\author{
F. K. Helms ${ }^{1}$; A. B. Loreto ${ }^{13 *} ;$ D. F. Adamatti2 ; C. Buss 3 ; A. L. A. Ferreira ${ }^{3}$ \\ ${ }^{I}$ Centro de Desenvolvimento Tecnológico, UFPel, 96010-210, Pelotas-RS, Brasil \\ 2Programa de Pós-graduação em Modelagem Computacional, FURG, 96203-900, Rio Grande-RS. Brasil \\ ${ }^{3}$ Programa de Pós-graduação Ensino de Ciência e Matemática, UFPel, 96010-210,Pelotas-RS, Brasil \\ *alineloreto@gmail.com
}

(Recebido em 22 de outubro de 2014; aceito em 07 de março de 2015)

\begin{abstract}
O presente trabalho tem como objetivo propor uma modelagem de agentes pedagógicos para desenvolver um sistema tutor baseado em multiagentes a fim de auxiliar na aprendizagem da Matemática no ensino médio. Mais especificamente, modela-se um tutor para auxiliar o aluno na resolução de exercícios para aprendizagem do conteúdo de equações algébricas. Com o desenvolvimento do sistema tutor multiagentes espera-se incentivar o estudo de Matemática, de forma lúdica, com auxílio de uma ferramenta computacional e reforçar os conteúdos da Matemática do Ensino Médio.

Palavras-chave: multiagentes, aprendizado, matemática.
\end{abstract}

This paper aims to propose a model of pedagogical agents to develop a tutoring system based on multiagent in order to assist in the learning of Mathematics in high school. More specifically, modeling is a tutor to help students in solving exercises for learning the content of algebraic equations. With the development of multi-agent system tutor is expected to encourage the study of Mathematics in a playful manner, with the aid of a computational tool and reinforce the content of Middle School Math.

Keywords: multiagent, learning, mathematics

\section{INTRODUÇÃO}

Segundo Moran [1], o uso das tecnologias propicia novos modos de produção, porém não altera a relação pedagógica e nem substitui o professor, estão disponíveis para que o professor use de modo a variar as práticas pedagógicas de algumas de suas aulas. Fazendo das tecnologias em suas ações docentes o professor pode tornar-se instigador da curiosidade do aluno, porém ele não deve deixar de contextualizar suas atividades adaptando-as ao meio que o aluno está inserido. Com o uso das tecnologias pode haver uma maior aproximação entre o professor e o aluno, pois pode existir uma comunicação via mensagens, o que pode contribuir para que o professor adapte suas aulas para desenvolver os conteúdos seguindo ou baseando-se no ritmo dos alunos. O bom uso das tecnologias na educação pode levar a um processo de ensino aprendizagem mais dinâmico e atrativo.

Alunos da Educação Básica, independente da classe social, possuem grandes dificuldades no aprendizado de Matemática, sendo a Álgebra um expoente entre tais assuntos [2]. Estudos mostram que os alunos brasileiros têm grande dificuldade em assuntos relacionados à Álgebra [3]. Nos resultados do Sistema Nacional de Avaliação da Educação Básica (SAEB), por exemplo, itens referentes à Álgebra raramente atingem um índice de $40 \%$ de acerto em muitas regiões do país. Inicialmente nos estudos matemáticos os alunos lidam com uma série de cálculos aritméticos, tendo como desafio realizar uma cadeia sucessiva de operações, com a devida ordem de resolução. Em um segundo momento, na Álgebra, é preciso fazer relações e simplificar termos, a fim de encontrar valores que solucionem uma condição proposta em uma base de dados estatísticos sobre cada aluno, o que auxiliará o professor na análise das deficiências individualmente e em grupo.

O principal objetivo é propor uma modelagem para agentes pedagógicos para desenvolver um sistema tutor baseado em multiagentes a fim de auxiliar na aprendizagem da Matemática no ensino médio. Mais especificamente, modela-se um tutor para auxiliar o aluno na resolução de 
exercícios de fixação de equações algébricas. Com o desenvolvimento do sistema tutor multiagentes espera-se incentivar o estudo de Matemática, de forma lúdica, com auxílio de uma ferramenta computacional e reforçar os conteúdos da Matemática do Ensino Médio como forma de avaliar os conhecimentos adquiridos.

\section{MATERIAL E MÉTODOS}

De acordo com Wooldridge [13], um agente é um sistema de computador que está situado em algum ambiente e que é capaz de executar ações autônomas de forma flexível neste ambiente, a fim de satisfazer seus objetivos de projeto. O termo "agente" é usado com frequência na Inteligência Artificial, mas não há um consenso para o que um agente é ou significa, podendo ser classificado como sendo desde simples processos de hardware e/ou software até entidades sofisticadas com capacidade de realizar tarefas complexas. Mais especificamente, "agente" pode ser entendido como uma entidade que exibe alguns aspectos da inteligência humana [14].

Agentes pedagógicos, segundo Giraffa [15], são aqueles utilizados em sistemas que utilizam o paradigma de agentes desenvolvidos para fins educacionais, podendo atuar como tutores virtuais, estudantes virtuais, ou ainda companheiros virtuais de aprendizagem, tendo como objetivo auxiliar os estudantes no processo de ensino-aprendizagem.

Sistemas Tutores Inteligentes (STI) são sistemas computadorizados capazes de ensinar conteúdos, através de Modelos Instrucionais que estejam presentes em sua arquitetura [4]. Segundo Viccari [16] são sistemas capazes de, através da interação com o usuário, atualizar sua própria base de conhecimento sobre o aluno (modelo de aluno). Jennings [17] define Sistemas Multiagentes (SMA) como uma rede de resolvedores de problemas que trabalham juntos para solucionar problemas que estão além das capacidades individuais de cada resolvedor de problema. Para desenvolver um sistema tutor baseado multiagentes para ensino de Matemática no ensino médio, conheceu-se primeiramente uma escola pública e a realidade do ensino de Matemática. Após contato com professores de Matemática, estabeleceu-se os conteúdos a serem implementados - equações algébricas - e na sequência, escolheu-se os exercícios a serem utilizados pelo tutor inteligente. Com o tutor inteligente desenvolvido deseja-se sistematizar as tarefas a serem desenvolvidas pelos alunos.

Com o objetivo que modelar um tutor inteligente baseado em multiagentes para auxiliar o aluno na resolução dos exercícios para aprendizagem do conteúdo de equações algébricas, propõe-se uma metodologia composta pelos seguintes componentes: Aluno, Interface, Tutor, Base de Informações de Equações e Base de Conhecimento/Planos com Dicas.

A metodologia proposta no presente trabalho é apresentada na Figura 1.

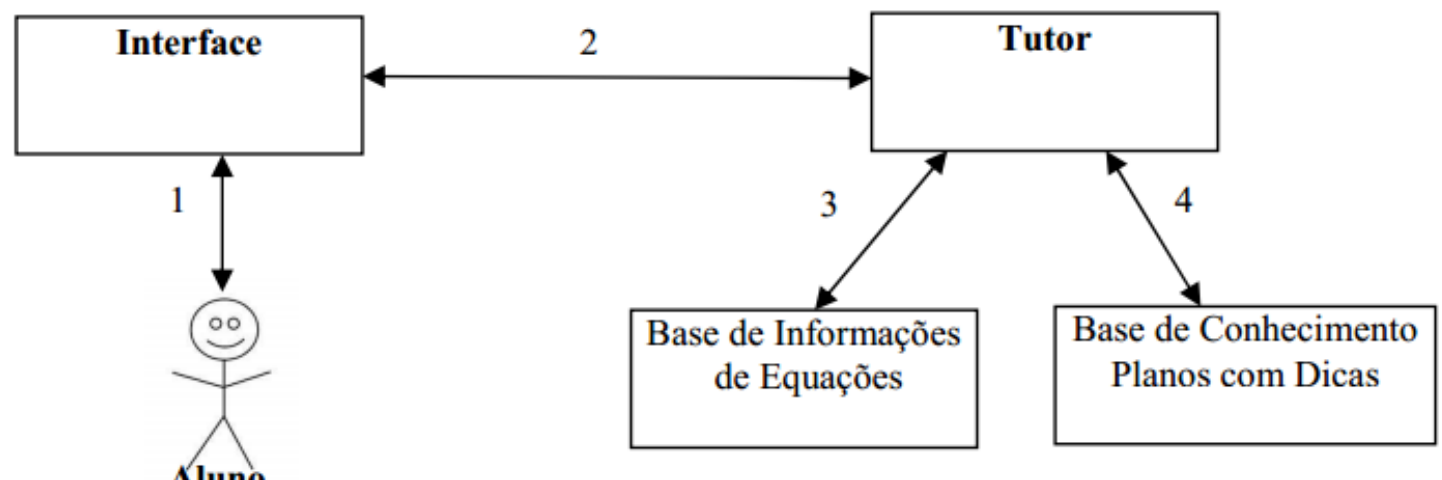

Figura 1: Metodologia do Tutor

A seguir descreve-se os seguintes componentes da metodologia proposta:

- Aluno: usuário do sistema;

- Interface: software de interação entre o Aluno e o tutor inteligente. A interface inicial proposta é apresentada na Figura 2; 


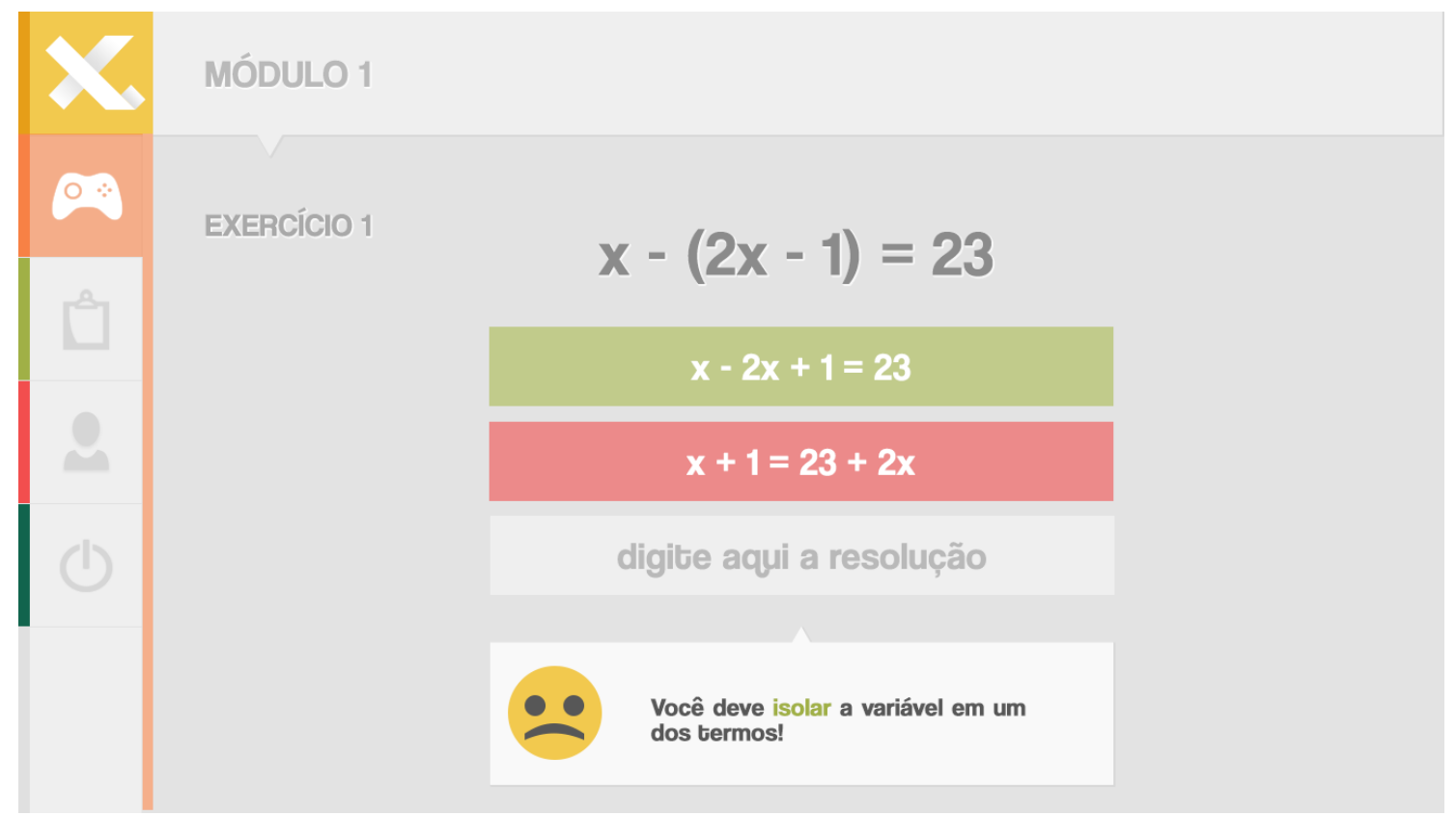

Figura 2: Interface do Tutor

- Tutor: software tutor inteligente multiagentes, recebe as informações da interface, de acordo com o que o Aluno informou, e "raciocina" se a resposta está correta pela Base de Informações de Equações ou se o aluno precisa de maiores orientações para dar continuidade as suas atividades pela Base de Conhecimento (conjunto de Planos com Dicas);

- Base de Informações de Equações: tem armazenadas todas as equações que podem ser desenvolvidas pelos Alunos, com suas soluções. É vital para saber se o Aluno concluiu a atividade e indicar a próxima equação a ser feita, seguindo um nível crescente de dificuldade;

- Base de Conhecimento: tem armazenados todos os planos que indicarão as dicas para os

Alunos conseguirem resolver as equações.

Propõe-se também no sistema sete Módulos conforme onível crescente de dificuldade. Estes Módulos são identificados conforme as cores de faixas do Karatê [20].

\section{RESULTADOS E DISCUSSÃO}

Como a intenção não é o ensino e aprendizado à distância, e sim um software que possa ser utilizado em sala de aula, ou laboratório de informática, ou ainda em casa para auxiliar o aluno na realização do tema, pensou-se em uma interface simples com agente inspirados nos emojis. Acredita-se que com esta aparência o agente fique próximo dos conhecidos emoticons, recorrentes na vida dos adolescentes em todas as redes sociais.

A Figura 3 destaca os componentes da interface proposta na Figura 2. 


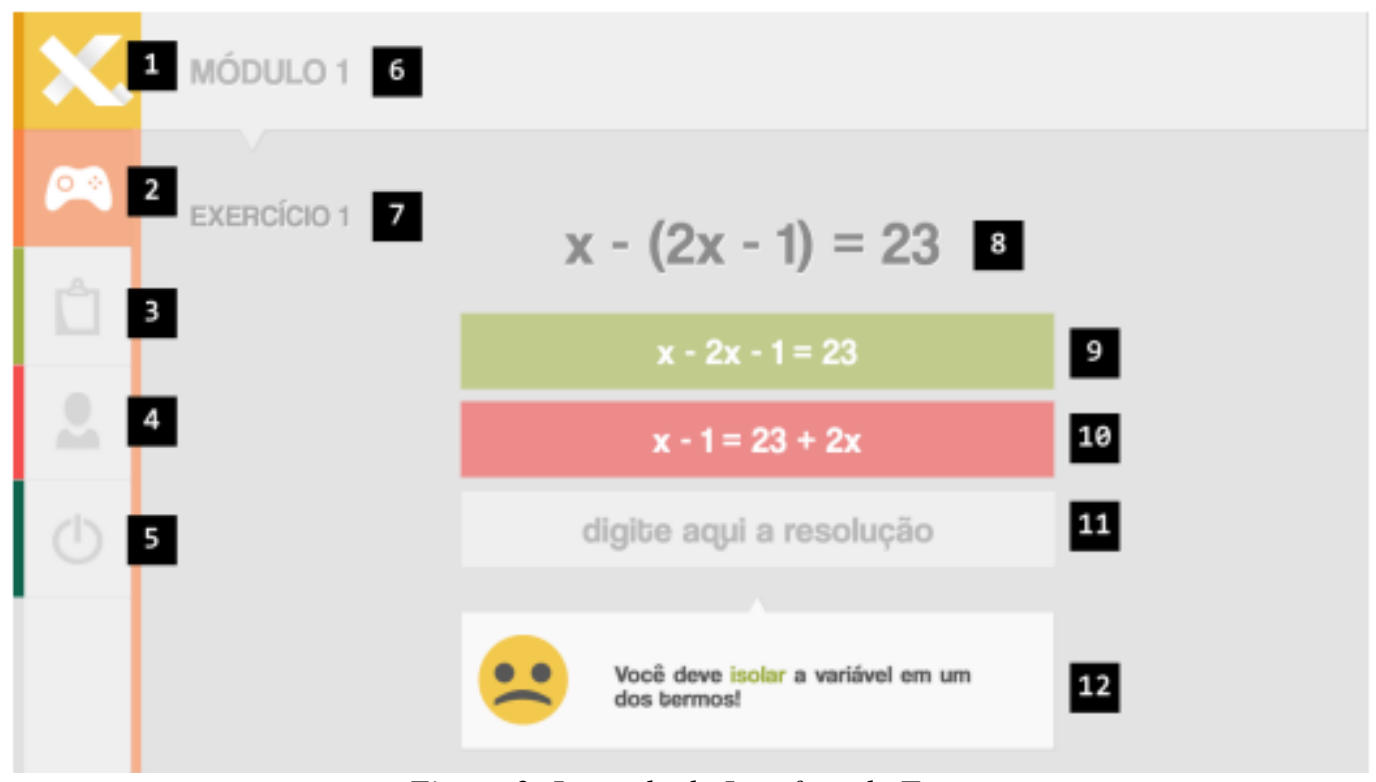

Figura 3: Legenda da Interface do Tutor

Legenda do protótipo da Interface:

1 - Logo do tutor: Conforme o aluno avança pelos módulos (no total são 7 módulos conforme o grau de dificuldade dos exercícios), o logo altera a sua cor seguindo as cores das faixas de karatê: amarelo, laranja, azul, verde, roxo, marrom e preto.

2 - Primeira opção do menu: painel de jogo, onde o aluno realiza os exercícios.

3 - Segunda opção do menu: painel de ajudas, onde estão todos os documentos no formato Pdf que o professor disponibilizar. Nota-se que a cor do menu é verde, assim como os links nas dicas e a cor representativa do acerto, fazendo a relação "você sabe o que está no conteúdo do professor, logo você acerta".

4 - Terceira opção do menu: painel do aluno, onde constam todas as informações como nome, número de tentativas por exercício, número de exercícios realizados e número de acessos no painel de ajudas.

5 - Quarta opção do menu: sair do tutor.

6 - Indica o módulo em que o aluno se encontra.

7 - Indica o número do exercício que o aluno está resolvendo.

8 - Equação que o aluno deve resolver.

9 - Quando o campo está verde, indica que o aluno acertou este passo.

10 - Quando o campo está vermelho, indica que o aluno errou este passo.

11- Campo neutro, o aluno não submeteu a resposta ainda.

12 - Caixa de dicas, onde o aluno recebe sugestões para corrigir o seu erro e incentivos para prosseguir com o exercício.

As cores do protótipo de interface do tutor foram cuidadosamente escolhidas para serem suaves e para estimular o usuário. Segundo Farina, Perez e Bastos [18], as cores constituem estímulos psicológicos para a sensibilidade humana, influindo no indivíduo, para gostar ou não de algo, para negar ou afirmar, para se abster ou agir. Ainda, segundo esses autores, o verde remete a esperança, perseverança e juventude; o vermelho estimula a conquista e o dinamismo; o amarelo desperta e simboliza a criatividade e juventude; e o laranja é uma cor estimulante.

$\mathrm{Na}$ elaboração da interface foram considerados Critérios Ergonômicos desenvolvidos pelos pesquisadores do INRA (Institut National de Recherche en Informatique et en Automatique) Christian Bastien e Dominique Scapin [19]. Os Critérios Ergonômicos são uma série de características que uma UI (User Interface) deve possuir. Neste caso, importante observar os seguintes critérios:

- Condução: apresenta-se na forma de rótulos, legendas ou alertas. Como o tutor é organizado hierarquicamente por meio de módulos, é importante informar em que módulo e exercício o 
usuário se encontra. Além disso, considerou-se um agrupamento e distinção de itens eficaz, separando módulos por cores e também relacionando as opções do menu de navegação com as ações do Tutor. Aliando isso à preocupação com a legibilidade da interface, o usuário não encontrará dificuldades em navegar e aprender com a mesma.

- Carga de trabalho: foi priorizada uma interface em que a densidade informacional fosse baixa, porém suficiente para o correto entendimento da mesma e da atividade proposta.

Consistência: como já citado no critério Condução, foi desenvolvido um padrão para atividades semelhantes em diferentes áreas da interface, no caso foram usadas predominantemente - cores.

$\mathrm{Na}$ Figura 3, pode-se verificar que existe uma ordem para execução das atividades. Primeiramente, o Aluno, via Interface, informa sua primeira solução para a equação. Em seguida, o Tutor verifica se a solução está correta (resposta final) ou se ainda não foi finalizada. No caso da não finalização existem duas possibilidades: estar correta, mas não finalizada; ou estar errada. O Tutor, a partir de uma análise sintática, deve detectar em que momento da resolução o Aluno se encontra. Ressalta-se que estamos propondo um sistema multiagentes cognitivo, possuindo uma Base de conhecimento que contém "planos de dicas" que são apresentadas ao Aluno conforme a resolução do exercício. Um exemplo de dica aos Alunos é apresentada na Figura 4.

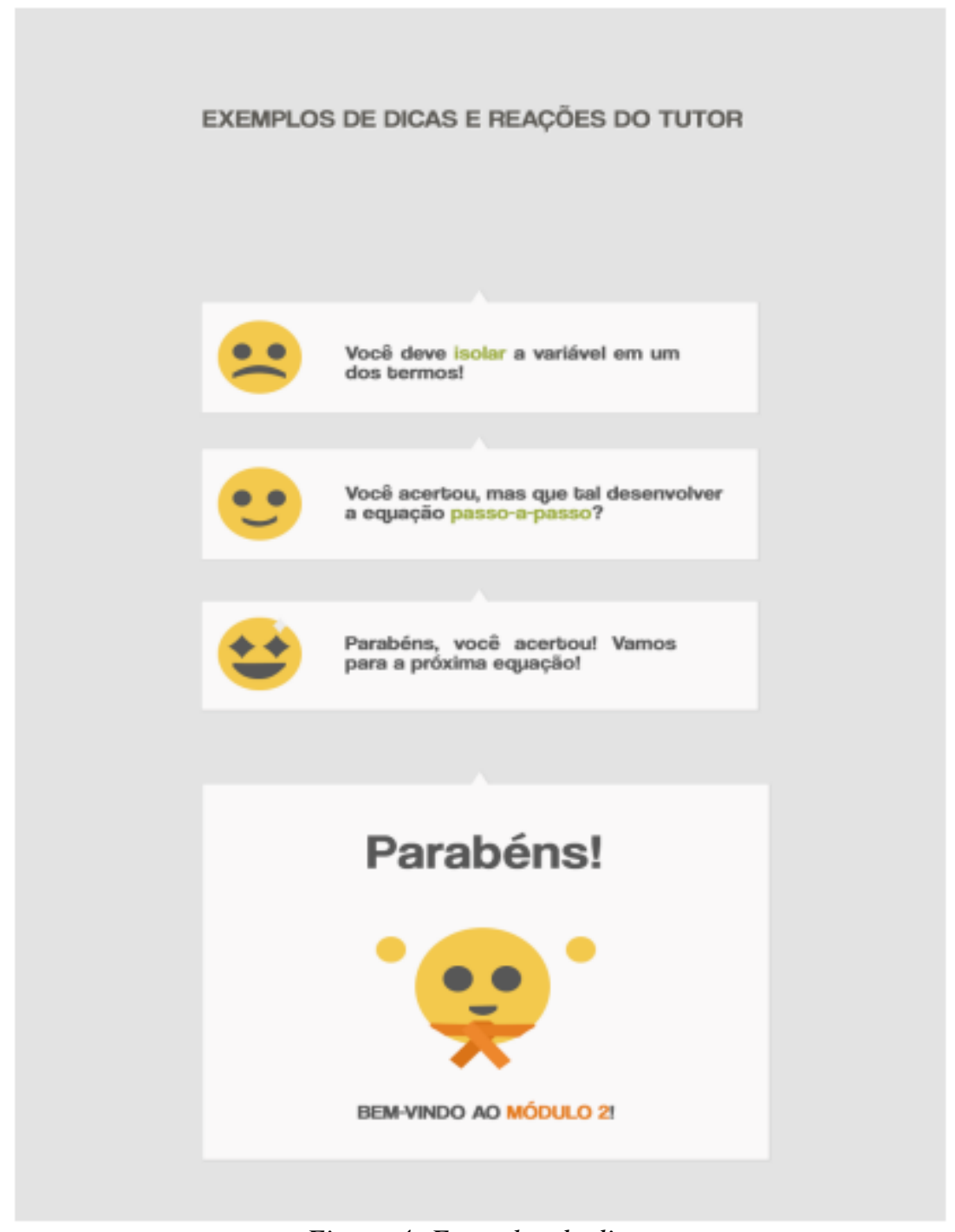

Figura 4: Exemplos de dicas

As reações do agente (Figura 5) foram inspiradas nos emojis, recorrentes na vida dos adolescentes em todas as redes sociais. 


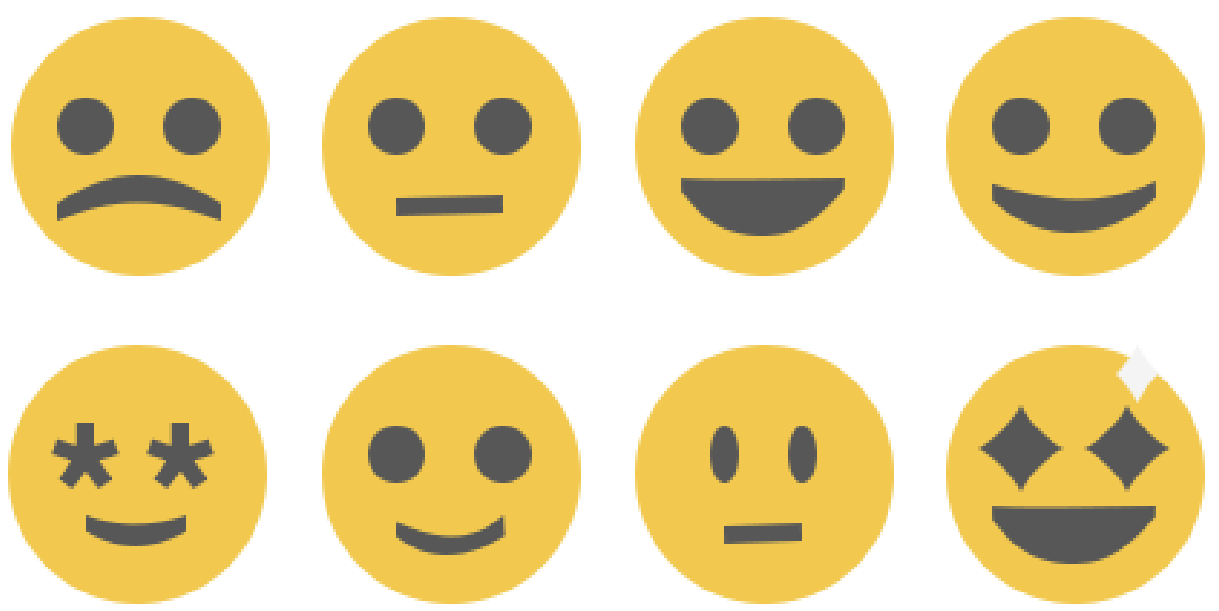

Figura 5: Agente com diferente grau de conhecimento

A cor e a classificação dos módulos do Tutor foram inspiradas nas faixas de Karatê. Segundo a Federação de Karatê-Dô Tradicional da Bahia [20] as cores relacionadas com o grau de conhecimento encontram-se na Tabela 1:

Tabela 1 - A classificação de cores das faixas de Karatê segundo a Federação de Karatê-Dô Tradicional da Bahia

\begin{tabular}{cc}
\hline Cores & Módulos do Tutor \\
\hline amarelo & Módulo 1 \\
vermelho & Módulo 2 \\
laranja & Módulo 3 \\
verde & Módulo 4 \\
roxo & Módulo 5 \\
marrom & Módulo 6 \\
preto & Módulo 7 \\
\hline
\end{tabular}

A medida que o aluno vai resolvendo os exercícios para aprendizagem e fixação do conteúdo de equações algébricas, o tutor vai disponibilizando exercícios com maior grau de dificuldade de resolução e o Agente vai evoluindo conforme as cores das faixas de Karatê (Figura 6).

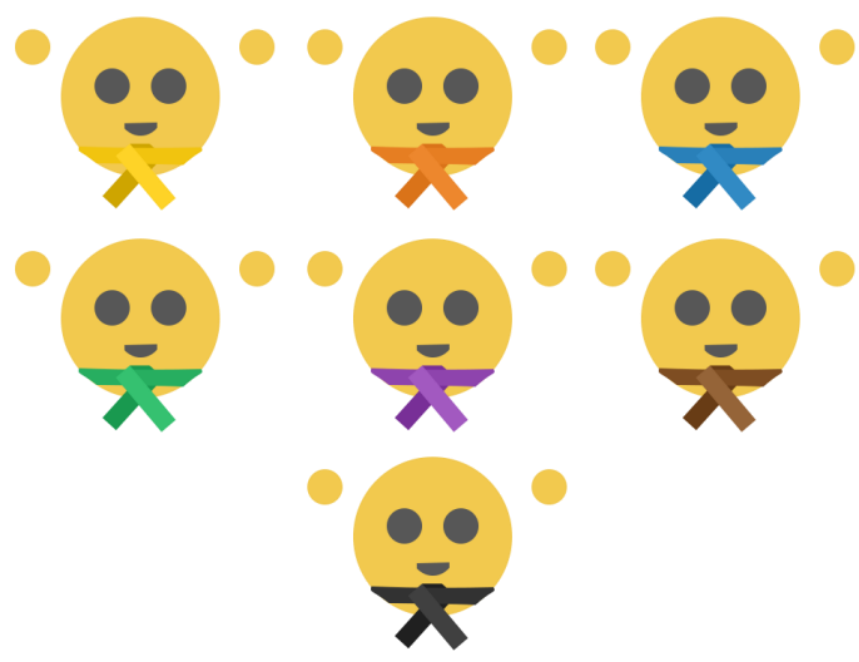

Figura 6: Evolução dos Módulos 


\section{CONCLUSÃO}

Espera-se que a integração de uma ferramenta computacional para ensino da Matemática no ensino básico desperte o interesse no aluno em aprender de forma lúdica. O modelo proposto não possui como objetivo substituir o professor da sala de aula, e sim oportunizar um software que auxilie o aluno na resolução de exercícios (especificamente equações algébricas).

Através do painel do usuário proposto no Tutor, onde constam todas as informações do usuário, como nome, número de tentativas por exercício, número de exercícios realizados e número de acessos no painel de ajudas o professor terá um retorno da aprendizagem do aluno referente aquele conteúdo. Possibilitando estratégias para reforçar o ensino e aprendizagem.

Nossa motivação é desenvolver recursos que permitam a integração de ferramentas computacionais, no caso desse projeto, um tutor inteligente, com conteúdos de Matemática que são abordados no Ensino Médio. A Matemática envolve os estudantes nos processos decisórios, coloca nas mãos dos professores um conhecimento que pode ser repassado no ciclo básico, despertando o interesse dos estudantes por essas áreas. Existe um grande potencial de disseminação deste trabalho em outras instituições de ensino, tanto em nível médio como em nível superior. O principal motivo para esse potencial de disseminação é que será desenvolvido um tutor inteligente, onde diversos exercícios poderão ser acessados e este material será disponibilizado gratuitamente na Web.

\section{AGRADECIMENTOS}

Os autores agradecem ao CNPQ- Chamada No 18/2013 MCTI/CNPq/SPM-PR/Petrobrás Meninas e Jovens Fazendo Ciências Exatas, Engenharias e Computação.

\section{REFERÊNCIAS BIBLIOGRÁFICAS}

1. Moran JM. Novas tecnologias e o re-encantamentodo mundo. Tecnologia Educacional. Rio de Janeiro. 1995 setembro-outubro;23(126):24-26.

2. Stacey K, Macgregor M. Learning the Algebraic Method of Solving Problems. Journal of Mathematical Behavior. 2000.

3. Brasil. Secretaria de Educação Fundamental. Parâmetros curriculares nacionais: ciências. MEC. Brasília, 1998. [atualizado em 12 nov 2013]. Disponível em: http://portal.mec.gov.br/seb/arquivos/pdf/ciencias.pdf

4. Murray T. Authoring intelligent tutoring systems: An analysis of the state of the art. International Journal of Artificial Intelligence in Education. 1999;10:98-129.

5. Bloom BS. The 2 Sigma Problem: The search for methods of group instruction as effective as one-toone tutoring. In: Educational Researcher. 1984, p. 4-16.

6. Anderson J et al. General principles for an intelligent tutoring architecture. In: Cognitive Approaches to Automated Instruction. Mawah, NJ: Erlbaum. 1992.

7. Jaques PA, Viccari RM. PAT: um agente pedagógico animado para interagir afetivamente com o aluno. Novas Tecnologias na Educação. 2005 Maio;3(1):1-19.

8. Mello G, Carlotto T, Rubi G, Seffrin HM, Jaques P. Implementando o Agente de Base de Domínio do Sistema Tutor Inteligente PAT2Math. Novas Tecnologias na Educação. 2009 Julho;7(1).

9. Bocca E, Jaques P, Viccari R. Modelagem e Implementação da Interface para Apresentação de Comportamentos Animados e Emotivos de um Agente Pedagógico Animado. Novas Tecnologias na Educação. 2003 setembro;1(2):1-14.

10. Soares VC, Rissoli VRV. Agente Inteligente no apoio Ensino-Aprendizagem, in Anais do XXII SBIE - XVII WIE 22 Simpósio Brasileiro de Informática na Educação (SBIE 2011). 2011. p. 180-183.

11. Oliveira PPB, Ferneda E, Prado HA, Bittencourt II. Integrando sistemas inteligentes a jogos, in Anais do XXIII SBIE - XVIII WIE 23º Simpósio Brasileiro de Informática na Educação (SBIE 2012). 2012. p. $1-10$.

12. Silva JH, Couto LS, Odakura V. Aparência de um agente pedagógico animado para um ambiente virtual de aprendizagem, in Anais do XXIIISBIE - XVIII WIE $23^{\circ}$ Simpósio Brasileiro de Informática na Educação (SBIE 2012). 2012. p. 1-5.

13. Wooldridge M. An Introduction to MultiAgent Systems. [S.1.]:Wiley. 2002. 
14. Damasceno FR. Concepção e Desenvolvimento do Agente Tutor e Modelo de Aluno no Ambiente Inteligente de Aprendizagem PAT2MATH. Dissertação de Mestrado em Computação Aplicada. Universidade do Vale dos Sinos. 2011; 155 p.

15. Giraffa LMM. Uma arquitetura de tutor utilizando estados mentais. Tese (Doutorado em Ciências da Computação) - Instituto de Informática, UFRGS, Porto Alegre. 1999.

16. Viccari RM. Um Tutor Inteligente para a programação em Lógica - Idealização, Projeto e Desenvolvimento. Universidade de Coimbra, Tese (Doutorado). 1990.

17. Jennings NR et al. A Roadmap of Agent Research and Development. Autonomous Agents and MultiAgent Systems, 1,p.275-306-Kluwer Academic Publisher, Boston. 1998.

18. Farina M, Perez C, Bastos D. Psicodinâmica das cores em comunicação, São Paulo, Editora Edgard Blücher Ltda. 5. ed. 2006.

19. Scapin D, Bastien C. Critérios Ergonômicos para Avaliação de Interfaces Homem-Computador, Disponível em: http://www.labiutil.inf.ufsc.br/CriteriosErgonomicos/Abertura.html. Acesso em: 22 jan. 2014

20.Karatê, Karatê-Faixas", Federação de Karatê-Dô Tradicional da Bahia, Disponível em: http://www.fktb.com.br/faixas.asp. Acesso em: 17/07/2014. 